\title{
Einzelbetriebliche Auswirkungen politischer Reformen in der Landwirtschaft: Erste empirische Erkenntnisse
}

\author{
Jan-Henning Feil, Oliver Mußhoff, Tobias Roeren-Wiemers
}

\begin{abstract}
Kernaussagen
Politikfolgenabschätzungen sind insbesondere im Agrarbereich, z.B. in Bezug auf die anstehenden Reformen der gemeinsamen EU-Agrarpolitik, hoch relevant. Für das Feld der Politikberatung stellt sich hierbei vor allem die Frage nach geeigneten und allgemein verwendbaren Methoden, um politische Entscheidungen adäquat zu unterstützen. In diesem Beitrag werden die Effekte der EU-Agrarrefomen der letzten 20 Jahre und der Einführung des ErneuerbareEnergien-Gesetzes auf die Höhe der Einkommen landwirtschaftlicher Betriebe, auf Überwälzungseffekte auf dem Pachtmarkt sowie auf das Einkommensrisiko erstmalig anhand einzelbetrieblicher Daten untersucht. Hierzu steht ein Datensatz von landwirtschaftlichen Betrieben aus Nordrhein-Westfalen über einen Zeitraum von 1984/85 bis 2010/11 zur Verfügung, der mit Hilfe einer Paneldatenanalyse ausgewertet wird. Die Ergebnisse zeigen unter anderem, dass die besagten Reformen einen signifikanten Einfluss auf die Höhe der betrieblichen Einkommen hatten. Durch die Untersuchung wird gleichzeitig ein Modellrahmen vorgeschlagen, der bei Vorliegen einzelbetrieblicher Daten beliebig reproduzierbar und somit auch auf zukünftige politische Reformen im Allgemeinen und Agrarreformen im Besonderen anwendbar ist.
\end{abstract}

\section{Einleitung}

Die politischen Rahmenbedingungen, unter denen landwirtschaftliche Betriebe in Europa und insbesondere in Deutschland produzieren, sind einem stetigen Reformprozess ausgesetzt. Bezüglich vergangener Reformen sind hierbei vor allem die Reformen der gemeinsamen EU-Agrarpolitik (GAP) und die Einführung des Erneuerbare-Energien-Gesetzes (EEG) zu nennen. Im Rahmen der EU-Agrarpolitik wurden durch die im Jahr 1992 beschlossene MacSharry Reform die Interventionspreise für landwirtschaftliche Produkte verringert und produktionsgebundene Direktzahlungen als Kompensation eingeführt. Mit der Agenda 2000 wurde eine weitere Senkung der Interventionspreise und im Gegenzug eine Erhöhung der Direktzahlungen beschlossen. Letztere wurden fortan nicht mehr ausschließlich an die Produktion, sondern zusätzlich an Bewirtschaftungsauflagen bei der Landschaftspflege und an die Einhaltung von Mindeststandards beim Umwelt-, Arbeitsund Tierschutz gekoppelt. Die Fischler-Reform im Jahr 2003 entwickelte die Änderungen der Agenda 2000 weiter, indem sie die Direktzahlungen weitestgehend von der Produktion entkoppelte und eine fixe Betriebsprämie pro förderfähigem Hektar einführte. Mit der Einführung des EEG wurde die bevorzugte Einspeisung von Strom aus erneuerbaren Quellen, z.B. Biomasse, Windkraft, Solar, ins Stromnetz geregelt und den Erzeugern feste Einspeisevergütungen garantiert.

Im Rahmen der anstehenden GAP-Reform in 2014 werden die finanziellen Zuwendungen an die Landwirtschaft abermals neu geordnet. So wird nur noch ein Teil der bisherigen fixen Betriebsprämie pro förderfähigem Hektar bestehen bleiben. Ergänzend dazu soll jeder Landwirt unter dem Stichwort „Greening“ eine Hektarzahlung für die Einhaltung bestimmter dem Klima- und Umweltschutz förderlicher Bewirtschaftungsmethoden, z.B. der Erhalt von Dauergrünland, die Diversifizierung der Anbaufrüchte und der Erhalt ökologischer Vorrangflächen, erhalten. Diese anstehenden Beschlüsse werden 6 Jahre Bestand haben, bis der Finanzrahmen der GAP im Jahr 2020 abermals neu geordnet werden soll.

Im Regelwerk der EU ist festgelegt, dass in vielen Politikfeldern, insbesondere denen der GAP sowie der ruralen Entwicklung, laufend Folgenabschätzungen von bereits umgesetzten sowie zukünftigen Reformen vorgenommen werden müssen (Europäische Kommission, 2005). Hierbei sollen die Verantwortlichkeiten politischer Entscheidungsträger sichergestellt und die Zielerreichung der Reformen gemessen werden. Für das Feld der Politikberatung stellt sich hierbei insbesondere die Frage nach geeigneten Methoden der Folgenabschätzung vergangener und zukünftiger Reformen unter Berücksichtigung der jeweiligen Reformziele der politischen Entscheidungsträger.

In Bezug auf die vergangenen Reformen im Agrarsektor werden seit einiger Zeit die Auswirkungen auf den ökonomischen Erfolg landwirtschaftlicher Unternehmen sowie auf Überwälzungseffekte - insbesondere auf dem Pachtmarkt - in der Literatur intensiv diskutiert (z.B. Chatzis, 1997; Daugbjerg und Swinbank, 2007; Kilian und Salhofer, 2009). Darüber hinaus werden die Effekte politischer Reformen auf Preisrisiken auf den Agrarmärkten untersucht - insbesondere auf den Getreidemärkten (z.B. Sckokai und Moro, 2006; 2009). Hieraus lässt sich die Frage ableiten, ob sich ein verändertes Preisrisiko auch in einem veränderten Einkommensrisiko für landwirtschaftliche Betriebe niederschlägt.

Wissenschaftliche Analysen haben sich bislang weitestgehend darauf konzentriert, die betreffenden Auswirkungen politischer Strukturbrüche mit Hilfe modellbasierter Simulationsrechnungen zu quantifizieren (z.B. Britz et al., 2012; 
Happe und Balmann, 2002; Kreins und Gömann, 2008). Dabei wird vielfach von der Verhaltensannahme eines vollständig informierten und ausschließlich gewinnmaximierenden homo oeconomicus ausgegangen. Reale Wirtschaftssubjekte verfolgen aber in aller Regel Mehrfachziele, die neben dem Gewinn- auch das Sicherheitsstreben sowie internalisierte Wertvorstellungen und weitere nicht-monetäre Motivationen (z.B. Tradition oder soziale Anerkennung) beinhalten (z.B. Benz, 2006). Zudem verhalten sie sich, zumindest teilweise, begrenzt rational (z.B. Simon, 1956). Deshalb besteht bei Politikfolgenabschätzungen, die auf Simulationsrechnungen basieren, die Gefahr, dass Art und Geschwindigkeit des Anpassungsverhaltens an veränderte Rahmenbedingungen falsch eingeschätzt werden. Umfangreichere empirische Untersuchungen der tatsächlichen einzelbetrieblichen Effekte veränderter politischer Rahmenbedingungen auf Basis einzelbetrieblicher Daten liegen bislang nicht vor.

Das Ziel dieses Beitrags besteht deshalb darin, die Auswirkungen der wichtigsten EU-Agrarreformen der letzten 20 Jahre - der MacSharry-Reform, der Agenda 2000 und der Fischler-Reform - sowie der Einführung des EEG auf einzelbetrieblicher Ebene empirisch zu untersuchen. Hierzu stehen Daten von 22 Marktfruchtbetrieben aus Nordrhein-Westfalen für den Zeitraum von 1984/85 bis 2010/11 zur Verfügung. Mittels einer Paneldatenanalyse werden die Effekte der potenziellen politischen Strukturbrüche auf die Höhe der betrieblichen Einkommen, der Pachtzahlungen sowie des Einkommensrisikos quantifiziert. Des Wissens der Autoren nach ist dies die erste empirische Analyse der Effekte der wichtigsten Agrarreformen der letzten beiden Jahrzehnte auf einzelbetrieblicher Ebene in Deutschland. Hierbei wird ein Modellrahmen vorgeschlagen, der bei Vorliegen einzelbetrieblicher Daten beliebig reproduzierbar ist. Er ist somit auch auf aktuelle Reformvorhaben im Agrarbereich, z.B. die anstehende GAP Reform in 2014, ex post anwendbar und kann somit Politikberatern als Instrument zur Unterstützung politischer Entscheidungsträger für zukünftige Reformen, z.B. der GAP Reform in 2020, dienen.

Der Beitrag ist wie folgt gegliedert: In Abschnitt 2 werden auf Grundlage bereits vorliegender Literatur die Forschungshypothesen abgeleitet. Nach der Charakterisierung der vorhandenen Daten in Abschnitt 3 folgt die Erläuterung zur methodischen Herangehensweise und der geschätzten Modelle in Abschnitt 4. Die Hypothesen aus Abschnitt 2 werden in Abschnitt 5 überprüft. Abschließend werden die Ergebnisse zusammengefasst und zukünftige Forschungsfelder aufgezeigt (Abschnitt 6).

\section{Hypothesengenerierung}

Im Folgenden werden drei Hypothesen aus der Literatur hergeleitet, die sich direkt auf die Einkommens- und Überwälzungseffekte der wichtigsten politischen Strukturbrüche der vergangenen 20 Jahre für landwirtschaftliche Betriebe beziehen. Diese Hypothesen sollen anschließend anhand einer
Paneldatenanalyse für den vorliegenden einzelbetrieblichen Datensatz getestet werden.

In Bezug auf die verschiedenen GAP-Reformen war es eines der erklärten Ziele der Politik, die Einkommenssituation landwirtschaftlicher Betriebe nicht zu verschlechtern. So versicherte die Europäische Kommission bereits vor der Einführung der MacSharry-Reform, dass die neu eingeführten Direktzahlungen die verringerten Preisstützungen in voller Höhe kompensieren und keine negativen Einkommenseffekte entstehen würden (Europäische Kommission, 1991). Wissenschaftliche Beiträge, die die (möglichen) Auswirkungen politischer Reformen auf die einzelbetriebliche Einkommenssituation anhand von Modellrechnungen quantifizieren, beziehen sich vor allem auf die Entkopplung der Prämien im Zuge der Fischler-Reform in 2003. Happe und Balmann (2002) kommen mit Hilfe des agentenbasierten Agrarstrukturmodells AgriPoliS zu dem Ergebnis, dass die Entkopplung der Direktzahlungen keinen bedeutenden Einfluss auf die Wettbewerbsfähigkeit und die Einkommenshöhe landwirtschaftlicher Betriebe hat. Isermeyer (2003) sieht in seiner Bewertung der (damals) vorläufigen Beschlüsse der Fischler Reform durch die Entkopplung tendenzielle Einkommensnachteile für kleine und mittlere Betriebe. Hingegen kommen Kreins und Gömann (2008) mit Hilfe des Agrar- und Umweltinformationssystems RAUMIS zu dem Schluss, dass viele Regionen Deutschlands im Zuge der Entkopplung eine Aufstockung ihres Direktzahlungsvolumens verzeichnen werden. In Verbindung mit weiteren Veränderungen der landwirtschaftlichen Rahmenbedingungen würde dies zu einem Einkommensanstieg in der Landwirtschaft führen (Kreins und Gömann, 2008: 204). In Bezug auf die Einführung des EEG im Jahr 2000 kommen Gömann et al. (2007) zu dem Ergebnis, dass die positiven Einkommenseffekte durch den Energiemaisanbau für die Landwirtschaft allenfalls moderat ausfallen. Zusammenfassend wird aus diesen teilweise gegensätzlichen Ergebnissen aus der Literatur folgende Hypothese abgeleitet:

H1(Einkommenseffekt): Durch die Reformschritte der EU und das EEG wurde die absolute Höhe der Einkommen landwirtschaftlicher Betriebe in Deutschland nicht signifikant beeinflusst.

Neben der Höhe der landwirtschaftlichen Einkommen werden in der Literatur die Auswirkungen politischer Reformen auf mögliche Überwälzungseffekte auf dem Pachtmarkt für Ackerland intensiv diskutiert. Ganz grundsätzlich stellt Chatzis (1997) in Bezug auf Flächenpachten heraus, dass Überwälzungseffekte durch politische Marktinterventionen entstehen und vor der MacSharry-Reform insbesondere durch die Höhe festgelegter Interventionspreise beeinflusst wurden. Im Zuge der Entkopplung der Direktzahlungen im Rahmen der Fischler-Reform geht Isermeyer (2003) von einem Absinken des gesamten Pachtpreisniveaus im Agrarsektor aus. Auch bei Ciaian et al. (2008) kommt es in unterschiedlichen Szenarien in der Regel zu veränderten Überwälzungseffekten. Happe und Balmann (2002) untersuchen ebenfalls mittels einer Szenarioanalyse die Auswirkungen der Agenda 2000 und der 
(zum damaligen Zeitpunkt noch nicht final beschlossenen) Fischler Reform auf den Pachtmarkt. Bei den von ihnen untersuchten Szenarien kommt es im Zeitverlauf zu einem leichten Sinken der Pachtpreise durch den Politikwechsel. Damit ergibt sich folgende Hypothese:

H2(Überwälzungseffekt): Durch die Reformschritte der EU werden Überwälzungseffekte auf den Pachtmärkten signifikant beeinflusst.

In den letzten Jahren werden in der Literatur vermehrt steigende Volatilitäten auf den Märkten für Agrarprodukte thematisiert (z.B. Tangermann, 2011). Viele Studien führen die Zunahme von Preisvolatilitäten primär auf die agrarpolitischen Reformen in der EU zurück, durch die der EU-Agrarsektor den Weltmärkten geöffnet wurde (z.B. Thompson et al., 2000; von Ledebur und Schmitz, 2011). Die Vermutung liegt nahe, dass sich die steigenden Preisschwankungen für Agrarprodukte letztlich auch in steigenden Einkommensschwankungen landwirtschaftlicher Betriebe widerspiegeln. Es lässt sich demnach folgende Hypothese ableiten:

H3(Einkommensrisiko): Durch die Reformschritte der EU werden die Einkommensschwankungen landwirtschaftlicher Betriebe signifikant beeinflusst.

\section{Datengrundlage}

Die für die Hypothesenüberprüfung verwendeten Daten wurden von einem nordrhein-westfälischen Arbeitskreis zur Verfügung gestellt. Die jährliche betriebswirtschaftliche Auswertung der Betriebe inklusive eines Betriebsvergleiches wurde von einem Berater vorgenommen. Für die Wirtschaftsjahre 1984/85 bis 2010/11 liegen einzelbetriebliche Daten von 22 Betrieben vor. Nach Definition des Testbetriebsnetzes des BMELVs sind alle Betriebe der Betriebsform „Ackerbau“ zuzuordnen. Das bedeutet, mehr als 2/3 der geldlichen Bruttoleistung entstammt dem Anbau von Getreide, Raps, Rüben oder sonstigen Marktfrüchten. Die betrachteten Betriebe weisen eine Flächenausstattung von durchschnittlich 177 ha im Betrachtungszeitraum auf. Auf dieser Fläche wurde von 1984/85 bis 2010/11 hauptsächlich Getreide und Raps angebaut, wobei 13 Betriebe zusätzlich Zuckerrüben in ihrem Anbauprogramm hatten.

Um Effekte der politischen Reformen auf die Höhe (H1) und die Schwankungen (H3) der betrieblichen Einkommen zu analysieren, muss eine betriebliche Erfolgsgröße betrachtet werden. Aus Gründen der Datenverfügbarkeit wird hierbei auf den durchschnittlichen jährlichen Deckungsbeitrag pro ha im Ackerbau inklusive der Direktzahlungen zurückgegriffen. Im vorhandenen Datensatz ist dieser definiert als Summe aller Marktleistungen plus der Direktzahlungen abzüglich der variablen Kosten für Saatgut, Dünger, Pflanzenschutz und Sonstiges (Pachten sind hierbei nicht berücksichtigt). Zur Quantifizierung der Änderung von Überwälzungseffekten (H2) werden die betriebsspezifischen durchschnittlichen jährlichen Pachtzahlungen pro ha herangezogen. Mit Blick auf die Deckungsbeiträge sind für jeden der 22 Betriebe für jedes der
27 Jahre Beobachtungswerte - insgesamt 594 - verfügbar. Für die Pachtzahlungen sind hingegen bei einigen Betrieben die Beobachtungen lückenhaft, so dass hier ein unbalancierter Paneldatensatz mit insgesamt 412 Beobachtungswerten vorliegt.

Die Politikfolgenabschätzung erfolgt im Rahmen einer Paneldatenanalyse. Hierbei wird über eine Dummy-Variable für den jeweiligen Zeitraum auf einen potenziellen Strukturbruch getestet. Als hauptsächliche Änderungen der politischen Rahmenbedingungen für landwirtschaftliche Betriebe in Deutschland sind im Betrachtungszeitraum die MacSharryReform in 1992, die Agenda 2000, die Einführung des EEG in 2000 sowie die Fischler-Reform in 2003 zu berücksichtigen. Für eine erste Übersicht wird der Betrachtungszeitraum von 1984/85 bis 2010/11 zunächst in drei Zeitabschnitte unterteilt: Der Zeitraum der hohen Interventionspreise vor der MacSharry Reform von 1984/85 bis 1992/93 („Intervention“), der Zeitraum zwischen der MacSharry Reform und der Agenda 2000 bzw. der Einführung des EEG von 1993/94 bis 1999/2000 („MacSharry“) sowie der Zeitraum von 2000/01 bis 2010/11 („Ag2000/Fischler/EEG“). Tabelle 1 gibt einen Überblick über die inflationsbereinigten ${ }^{1}$ minimalen, durchschnittlichen und maximalen Deckungsbeiträge, Pachtzahlungen sowie Standardabweichungen der Deckungsbeiträge dieser Zeitabschnitte.

Tabelle 1. Beschreibung der Zeitreihen (in €/ha)

\begin{tabular}{|c|c|c|c|c|}
\hline & & $\begin{array}{l}\text { Intervention } \\
(1984 / 85-1992 / 93)\end{array}$ & $\begin{array}{l}\text { MacSharry } \\
(1993 / 94-1999 / 00)\end{array}$ & $\begin{array}{l}\text { Ag2000/Fischler/EEG } \\
(2000 / 01-2010 / 11)\end{array}$ \\
\hline \multirow{3}{*}{ Deckungsbeitrag } & Minimuma) & 1375 & 1141 & 1147 \\
\hline & Durchschnitt ${ }^{\text {b) }}$ & 1997 & 1506 & 1502 \\
\hline & Maximum $^{\text {a) }}$ & 2649 & 2280 & 2582 \\
\hline \multirow{3}{*}{ Pachtzahlung } & Minimum ${ }^{\mathrm{a})}$ & 190 & 246 & 243 \\
\hline & Durchschnitt) & 431 & 483 & 437 \\
\hline & Maximum $^{\text {a) }}$ & 779 & 941 & 835 \\
\hline \multirow{3}{*}{$\begin{array}{l}\text { Standardabweichung } \\
\text { des Deckungsbeitrags }\end{array}$} & Minimum $^{\text {a) }}$ & 133 & 75 & 166 \\
\hline & Durchschnitt) & 305 & 163 & 242 \\
\hline & Maximum $^{\text {a) }}$ & 470 & 338 & 322 \\
\hline
\end{tabular}

a) Geringster bzw. höchster betriebsspezifischer Mittelwert im betrachteten Zeitraum

b) Mittelwert aller Betriebe im betrachteten Zeitraum

Mit Blick auf Tabelle 1 wird deutlich, dass der durchschnittliche Deckungsbeitrag im Zeitablauf gesunken ist. Die durchschnittliche Pachtzahlung pro ha lag nur im zweiten Zeitabschnitt höher. Schließlich war durch die deutlich reduzierte mittlere Standardabweichung des Deckungsbeitrags ein potentiell verringertes Einkommensrisikos nach der MacSharry-Reform zu verzeichnen. Die Standardabweichung stieg jedoch im darauf folgenden Zeitabschnitt wieder an. Ob diese $\mathrm{zu}$ beobachteten Entwicklungen in Bezug auf die einzelbetriebliche Einkommenshöhe, Überwälzungseffekte und das Einkommensrisiko signifikant sind, soll die im Folgenden dargestellte Paneldatenanalyse näher beleuchten.

1 Die Inflationsrate der Jahre 1985 bis 2011 lag zwischen -1,00\% und 4,22 \% (Bundesbank, 2012). Die um Inflation bereinigten Zeitreihen beziehen sich auf das Jahr 2012. 


\section{Methodik}

Zur Auswertung von Paneldaten stehen mehrere Modelle zur Verfügung (z.B. Baltalgi, 2009; Hsiao, 2003; Wooldridge, 2010). Ein einfaches gepooltes Modell kann wie folgt beschrieben werden (z.B. Baltagi, 2009: 12):

$$
\text { (1) } y_{i, t}=\alpha+\sum_{k=1}^{K} \beta^{k} x_{i, t}^{k}+\varepsilon_{i, t} \text { mit } i=1, \ldots, N ; t=1, \ldots, T \text { und } \varepsilon_{i, t} \sim \mathrm{N}\left(0, \sigma_{\eta}^{2}\right)
$$

Wobei $y_{i, t}$ die abhängige Variable und $x_{i, t}^{k}$ die k-te unabhängigen Variable des Individuums $i$ zum Zeitpunkt $t$ darstellt, $\alpha$ ist eine Konstante, $\beta^{k}$ der Parameter der k-ten unabhängigen Variable und $\varepsilon_{i, t}$ den Fehlerterm, für den angenommen wird, dass er unabhängig und identisch normalverteilt ist über $i$ und $t$ mit einem Erwartungswert von Null und einer Varianz von $\sigma_{\varepsilon}^{2}$.

Als abhängige Variable wird zur Untersuchung von H1 (Einkommenseffekt) für den vorliegenden Datensatz der jährliche ackerbauliche Deckungsbeitrag $D B_{i, t}$ in $€$ pro ha verwendet, der die ab dem Jahr 1993 gezahlte flächenspezifische Betriebsprämie beinhaltet. Für die Analyse der Überwälzungseffekte auf dem Pachtmarkt (H2) wird die individuell gezahlte jährliche Pacht $P a_{i, t}$ in $€$ pro ha gewählt. Um die Veränderung des Einkommensrisikos (H3) zu untersuchen, werden - in Anlehnung an die grundsätzliche Vorgehensweise von Minimization of Total Absolute Deviations- bzw. MOTADModellen (z.B. Hazell, 1971) - die absoluten Abweichungen der Deckungsbeiträge $\left|D B_{i, t}-D B_{i, t-1}\right|$ herangezogen. Der Einfluss der politischen Reformen wird über eine Dummy-Variable für die MacSharry-Reform (Wirtschaftsjahr 1993/94) sowie eine gemeinsame Dummy-Variable für die Agenda 2000, Einführung des EEG und die Fischler-Reform (Wirtschaftsjahr 2000/01) abgebildet. ${ }^{2}$

Außerdem werden Kontrollvariablen berücksichtigt: Skaleneffekte haben einen bedeutenden Einfluss auf den Erfolg von Unternehmen (z.B. Christensen und Greene, 1976). Für die vorliegende Untersuchung wird somit die Betriebsgröße in ha auf ihren Erklärungsgehalt hin untersucht. Des Weiteren verweisen wissenschaftliche Untersuchungen auf die Bedeutung der Kapitalintensität für den unternehmerischen Erfolg (z.B. Morck, 2000). Folglich wird die Kapitalausstattung pro ha, verstanden als Summe von Gebäude-, Maschinen- und Umlaufkapital, ebenfalls als erklärende Variable in das Modell aufgenommen. Außerdem wird in der agrarökonomischen Literatur vielfach der Einfluss des Zuckerrübenanbaus auf betriebliche Einkommen, Überwälzungseffekte und Einkommensschwankungen diskutiert (z.B. Schmidt, 2005). Unter Berücksichtigung der Tatsache, dass einige der vorliegenden Betriebe über den Betrachtungszeitraum Zuckerrüben anbauten und andere nicht, geht der Rübenanbau als unabhängige Variable in Form einer Dummy-Variable in das Modell ein. Weiterhin wird ein Zeittrend als erklärende Variable in das Modell aufgenommen. Hierdurch sollen allgemeine Effekte in den Preisen für landwirtschaftliche Produkte oder auch technischer Fortschritt berücksichtigt werden.
Grundsätzlich ist es nicht möglich, alle Variablen im Modell mit zu berücksichtigen, die einen Einfluss auf die abhängige Variable haben. Dies liegt zum einen daran, dass viele Einflussfaktoren nicht beobachtbar sind oder schlicht nicht mit erhoben wurden. In diesen Fällen besteht unbeobachtete Heterogenität, die in den Fehlerterm in Gleichung (1) einfließt. Dieser setzt sich dadurch wie folgt zusammen (z.B. Baltagi, 2009: 12):

$$
\text { (2) } \varepsilon_{i, t}=\mu_{i}+\eta_{i, t}
$$

Hierbei bezeichnet $\mu_{i}$ einen individuellen Fehler und $\eta_{i, t}$ einen allgemeinen Fehler. Sofern unbeobachtete Heterogenität besteht, sind die geschätzten Koeffizienten gemäß Gleichung (1) nicht mehr effizient, da $\varepsilon_{i, t}$ noch einen Erklärungsgehalt in Bezug auf die zu erklärende Variable in Höhe von $\mu_{i}$ hat. Für den vorhandenen Datensatz mit den beschriebenen Variablen zeigt ein nach Breusch und Pagan (1979) entwickeltes Testverfahren, dass unbeobachtete Heterogenität vorliegt. Um die unbeobachtete Heterogenität abzubilden, stehen zwei Ansätze zur Verfügung: das Fixed-Effects- und das Random-EffectsModell (FE-Modell und RE-Modell). Die Entscheidung für eines der beiden Modelle kann anhand eines nach Hausmann (1978) entwickelten Tests zur Spezifikation von Panelmodellen erfolgen. Wenn die individuellen und zeitlichen Fehler $\mu_{i}$ mit den unabhängigen Variablen $x_{i, t}^{k}$ nicht korreliert sind, kann durch die Schätzung eines RE-Modells die Schätzeffizienz erhöht werden. Besteht jedoch eine solche Korrelation, verliert der RE-Schätzer seine Konsistenz und es ist ein FEModell zu verwenden. Bei einem FE-Modell wird für die unbeobachtete Heterogenität kontrolliert, indem für jedes Individuum bzw. für jeden Betrieb eine Dummy-Variable ins Modell aufgenommen wird. Dadurch kann $\mu_{i}$ aus dem zusammengesetzten Fehlerterm eliminiert werden und die oben genannten Annahmen zum Fehlerterm wieder Gültigkeit erlangen. Für den vorliegenden Datensatz ist gemäß Hausmann-Test (Hausmann, 1978) ein FE-Modell zu verwenden. Das FE-Modell hat die Form (z.B. Baltagi, 2009: 13):

$$
\text { (3) } y_{i, t}=\mu_{i}+\sum_{k=1}^{K} \beta^{k} x_{i, t}^{k}+\eta_{i, t} \text { mit } \eta_{i, t} \sim \mathrm{N}\left(0, \sigma_{\eta}^{2}\right)
$$

Um das Problem einer möglichen Verzerrung der Koeffizienten der vorliegenden unabhängigen Variablen durch Heteroskedastizität und Autokorrelation zu vermeiden, werden bei der Auswertung robuste Standardfehler nach Arellano (1987) verwendet.

\footnotetext{
2 Zum Wirtschaftsjahr 2000/01 traten lediglich die Reformbeschlüsse der Agenda 2000 auf EU-Ebene sowie das EEG in Deutschland in Kraft. Die im Rahmen der Fischler Reform beschlossene Entkopplung der Direktzahlungen wurde erst zum Jahr 2005 deutschlandweit implementiert. Es wird jedoch im Rahmen der Untersuchung von einer zusätzlichen, dritten Dummy-Variable in 2005/06 abgesehen, da der Zeitraum zwischen 2000/01 und 2005/06 für eine aussagekräftige ökonometrische Auswertung zu kurz erscheint.
} 


\section{Ergebnisdiskussion}

Für den Test der drei Hypothesen werden im Folgenden die Ergebnisse der Paneldatenanalyse dargestellt und diskutiert. Die Konstante gibt hierbei aus Gründen der Übersichtlichkeit den Durchschnitt der betriebsindividuellen Konstanten aller 22 Betriebe wieder.

Tabelle 2. Ergebnisse des FE-Modells zur Überprüfung von $\mathrm{H}_{1}$ (Einkommenseffekt) mit dem ackerbaulichen Deckungsbeitrag als abhängige Variable

\begin{tabular}{lrrc}
\hline Variable & Koeffizient & $\mathrm{t}$-Wert & $\mathrm{p}$-Wert \\
\hline Konstante & 1913,170 & 19,800 & $<0,001 * * * *$ \\
Betriebsgröße & $-0,070$ & $-0,258$ & 0,796 \\
Kapitalausstattung & 0,022 & 2,633 & $0,009 * * *$ \\
Rübenanbau & 71,836 & 0,658 & 0,511 \\
Zeittrend & $-16,667$ & $-3,806$ & $<0,001 * * * *$ \\
MacSharry & $-410,355$ & $-10,530$ & $<0,001 * * *$ \\
Ag2000/Fischler/EEG & 120,260 & 3,259 & $<0,001 * * * *$ \\
\hline
\end{tabular}

594 Beobachtungen mit 22 Individuen, ${ }^{* * *}$ Irrtumswahrscheinlichkeit = 1\%, ${ }^{* *}$ Irrtumswahrscheinlichkeit $=5 \%,{ }^{*}$ Irrtumswahrscheinlichkeit $=10 \%, R^{2}$ $=0,687$

In Tabelle 2 sind die Ergebnisse zu H1 (Einkommenseffekt) dargestellt. Dabei wird ersichtlich, dass Änderungen in der Betriebsgröße offensichtlich keinen signifikanten Einfluss auf die absolute Höhe des Deckungsbeitrags pro ha eines Betriebes hatten. ${ }^{3}$ Dies deutet darauf hin, dass in den untersuchten Marktfruchtbetrieben keine bzw. nur sehr begrenzt Skaleneffekte erzielt werden konnten. Auch die Tatsache, dass ein Betrieb über den Betrachtungszeitraum mit Rübenanbau startete, resultierte nicht in einem signifikant höheren Deckungsbeitrag. Hingegen führte eine zunehmende Kapitalausstattung pro ha über den Betrachtungszeitraum zu einem signifikanten Anstieg des Deckungsbeitrags. Des Weiteren weist die Höhe der einzelbetrieblichen Deckungsbeiträge einen signifikant negativen Zeittrend auf.

Im Rahmen der Paneldatenanalyse beschreiben die Variablen MacSharry und Ag2000/Fischler/EEG zwei potentielle Strukturbrüche. Das Ergebnis für die Dummy-Variable der MacSharry-Reform deutet darauf hin, dass die vorgenommene stufenweise Reduzierung der Interventionspreise und gleichzeitige Einführung gekoppelter Direktzahlungen ab dem Jahr 1993 eine signifikante Reduzierung des ackerbaulichen Deckungsbeitrages um ca. $410 €$ pro ha und Jahr bedingte. Hingegen führten die Agenda 2000, die Fischler Reform sowie die Einführung des EEG im Vergleich zur MacSharry Reform zu einem signifikanten Anstieg des Deckungsbeitrages um ca. $120 €$ pro ha. Letzterer könnte unter anderem durch die preiserhöhenden Effekte des sich ausweitenden Energiemaisanbaus für andere Agrarrohstoffe infolge der Einführung des EEGs verursacht worden sein (vgl. Gömann et al., 2007). Zusätzlich muss jedoch beachtet werden, dass es weitere Markteinschnitte seit der Jahrtausendwende gab, die einen potenziellen indirekten Einfluss über den Preis auf die betriebliche Einkommenssituation gehabt haben könnten. Die OECD (2010) weist vor allem auf den weltweiten Abbau von Lagerbeständen für Agrarrohstoffe sowie das zuvor nicht vorhandene Engagement großer institutioneller Investoren an Warenterminbörsen hin.

Zusammenfassend zeigen die Ergebnisse des vorliegenden Modells, dass in den Jahren 1993/94 sowie 2000/01 zwei Strukturbrüche in der Entwicklung der einzelbetrieblichen Einkommen vorliegen, die auf politische Reformen zurückzuführen sein könnten. So wurden die ackerbaulichen Deckungsbeiträge der vorliegenden Betriebe einerseits durch die MacSharry Reform signifikant negativ und andererseits durch die Agenda 2000, die Fischler Reform und das EEG signifikant positiv beeinflusst. Die aufgestellte Hypothese H1 der unveränderten Einkommensentwicklung durch politische Reformen kann somit abgelehnt werden. Alternativ zum Jahr 2000/01 wurde für den zweiten Strukturbruch die Dummy-Variable Ag2000/Fischler/EEG erst in 2005/06 eingeführt, da erst zu diesem Zeitpunkt die Entkopplung der Direktzahlungen im Zuge der Fischler Reform deutschlandweit implementiert wurde (vgl. Fußnote 2). Dies führte zu qualitativ gleichwertigen Ergebnissen.

Tabelle 3. Ergebnisse des FE-Modells zur Überprüfung von $\mathrm{H}_{2}$ (Überwälzungseffekt) mit der durchschnittlich gezahlten Pacht als abhängige Variable

\begin{tabular}{lccc}
\hline Variable & Koeffizient & $\mathrm{t}$-Wert & p-Wert \\
\hline Konstante & 427,584 & 14,050 & $<0,001 * * * *$ \\
Betriebsgröße & $-0,441$ & $-4,903$ & $<0,001 * * *$ \\
Kapitalausstattung & 0,001 & 0,314 & 0,753 \\
Rübenanbau & 112,698 & 6,578 & $<0,001 * * * *$ \\
Zeittrend & $-4,630$ & $-1,607$ & 0,109 \\
MacSharry & 87,603 & 1,873 & $0,062 *$ \\
Ag2000/Fischler/EEG & 15,225 & 0,656 & 0,512 \\
\hline
\end{tabular}

412 Beobachtungen mit 22 Individuen, ${ }^{* * *}$ Irrtumswahrscheinlichkeit = 1\%,
Irrtumswahrscheinlichkeit $=5 \%,{ }^{*}$ Irrtumswahrscheinlichkeit = $10 \%, R^{2}$
$=0,713$

In Tabelle 3 sind die Ergebnisse zu H2 (Überwälzungseffekt) dargestellt. Die Ergebnisse zeigen, dass Betriebe signifikant geringere Pachtpreise zahlten, je mehr ihre Flächenausstattung im Betrachtungszeitraum anstieg. Diese Beobachtung könnte generell auf einen geringeren Anreiz für Betriebe hindeuten, höhere Pachten zu zahlen, welche bereits in den Vorjahren verstärkt Fläche hinzugewonnen haben. Im Gegensatz zu Betrieben mit lediglich Getreide und Raps, zahlten Betriebe, die während des Betrachtungszeitraums zusätzlich mit dem Anbau von Zuckerrüben starteten, signifikant höhere Pachtentgelte. Dies könnte mit einer höheren Profitabilität von Zuckerrüben gegenüber Getreide und Raps erklärt werden.

Obwohl durch die MacSharry Reform die Einkommensentwicklung der betrachteten Betriebe negativ beeinflusst wurde (siehe H1), stiegen die Pachtzahlungen in dem Zeit-

3 Es muss hierbei beachtet werden, dass durch die Verwendung eines FE-Modells lediglich Within-Effekte berücksichtigt werden können, d.h. das Modell kann im vorliegenden Fall nicht zwischen kleinen und großen Betrieben unterscheiden; geschätzt wird lediglich der Effekt der Größenänderung eines Betriebes im Betrachtungszeitraum. 
raum nach dieser Reform signifikant um ca. $88 €$ pro ha an. Diese Erkenntnis bestätigt die Argumentation von Chatzis (1997), der eine Reduzierung von Pachtpreisen unter die im Durchschnitt der EU gezahlten Beihilfen ausschließt. Flächeneigentümer bekamen auch ohne eine Bewirtschaftung der eigenen Flächen durch die MacSharry-Reform einen Teil der Betriebsprämien. Die Agenda 2000, die Fischler Reform und die Einführung des EEG führten hingegen zu keiner signifikanten Veränderung der Pachtpreise. Eine von Experten vorausgesagte signifikante Senkung von Pachtzahlungen durch die Agenda 2000 und die Fischler Reform kann also nicht nachgewiesen werden. Eine Ursache hierfür könnten verstärkte Investitionen in die flächenabhängige Tierhaltung ab diesem Zeitpunkt sein. Zusätzlich müssen auch die pachtpreiserhöhenden Effekte eines verstärkten finanziellen Engagements außerlandwirtschaftlicher Investoren im Agrarsektor berücksichtigt werden (OECD, 2010).

Zusammenfassend zeigen die Ergebnisse, dass im Jahr 1993/94 ein Strukturbruch in der Höhe der Pachtzahlungen vorlag und somit Überwälzungseffekte auf dem Pachtmarkt durch die MacSharry Reform signifikant beeinflusst wurden. H2 kann somit nicht abgelehnt werden. Analog zur Überprüfung von H1 wurde das Jahr 2005/06, alternativ zum Jahr 2000/01, auf einen Strukturbruch in den Pachtzahlungen hin überprüft. In diesem Falle konnte weder für das Jahr 1993/94 noch für das Jahr 2005/2006 ein signifikanter Strukturbruch verzeichnet werden.

Tabelle 4. Ergebnisse des FE-Modells zur Überprüfung von $\mathrm{H}_{3}$ (Einkommensrisiko) mit den absoluten Abweichungen der Deckungsbeiträge als abhängige Variable

\begin{tabular}{lccc}
\hline Variable & Koeffizient & $\mathrm{t}$-Wert & $\mathrm{p}$-Wert \\
\hline Konstante & 368,196 & 5,839 & $<0,001$ **** \\
Betriebsgröße & 0,137 & 1,532 & 0,126 \\
Kapitalausstattung & $-0,001$ & $-0,369$ & 0,712 \\
Rübenanbau & $-220,012$ & $-5,352$ & $<0,001$ **** \\
Zeittrend & 15,962 & 3,180 & $0,002 * * *$ \\
MacSharry & $-268,032$ & $-6,623$ & $<0,001$ **** \\
Ag2000/Fischler/EEG & $-56,791$ & $-1,239$ & 0,216 \\
\hline
\end{tabular}

571 Beobachtungen mit 22 Individuen, ${ }^{* * *}$ Irrtumswahrscheinlichkeit = 1\%, ${ }^{* *}$ Irrtumswahrscheinlichkeit $=5 \%,{ }^{*}$ Irrtumswahrscheinlichkeit $=10 \%, R^{2}$ $=0,127$

In Tabelle 4 sind die Ergebnisse zu H3 (Einkommensrisiko) dargestellt. Bei der Verwendung der absoluten Abweichungen der Deckungsbeiträge als abhängige Variable ist das Bestimmtheitsmaß mit $R^{2}=0,127$ erwartungsgemäß vergleichsweise gering. Für den Fall, dass ein Betrieb über den Betrachtungszeitraum mit dem Zuckerrübenanbau startete, verursachte dies eine signifikante Verringerung der absoluten Abweichungen der Deckungsbeiträge. Auch ist ein signifikanter positiver Zeittrend zu verzeichnen; das heißt, dass die Schwankungen der Deckungsbeiträge über den gesamten Betrachtungszeitraum zunahmen. Gründe hierfür könnten in den allgemein ansteigenden Preisvolatilitäten auf den Agrarmärkten ab diesem Zeitpunkt bestehen. Diese waren nicht nur durch eine Veränderung der politischen Rahmenbedingungen, sondern vor allem auch durch eine fortschreitende Öffnung der Märkte im Zuge der Globalisierung bedingt (OECD, 2010).

Der Koeffizient für die Dummy-Variable MacSharry deutet darauf hin, dass diese einen stabilisierenden Einfluss auf die betrieblichen Einkommen hatte: Die jährlichen absoluten Abweichungen der Deckungsbeiträge verringerten sich ab dem Jahr 1993/94 signifikant um ca. $268 €$ pro ha. Es ist zu vermuten, dass die Erlösschwankungen nach der Absenkung der Interventionspreise im Zuge der MacSharry-Reform ceteris paribus angestiegen sind. Preisschwankungen auf der Kostenseite, z.B. für Dünger und Saatgut, waren hingegen sowohl vor als auch nach der MacSharry Reform vergleichsweise hoch, da es hier keine staatlichen Preiseingriffe gab. Nach Abschaffung der Preisstützungssysteme auf der Erlösseite könnte ein natürlicher Hedge von Input- und Outputpreisen gewirkt haben, so dass Einkommensschwankungen verringert wurden. Wie die Ergebnisse für die Dummy-Variable Ag2000/Fischler/EEG zeigen, war ab dem Jahr 2000/01 keine zusätzliche signifikante Veränderung der absoluten Abweichungen der Deckungsbeiträge zu verzeichnen, die durch die betreffenden Reformen hätten verursacht werden können.

Zusammenfassend zeigen die Ergebnisse, dass im Jahr 1993/94 ein Strukturbruch in den absoluten Abweichungen der Deckungsbeiträge vorlag, der durch die MacSharryReform ausgelöst wurde. Dementsprechend kann H3, welche auf ein sich veränderndes Einkommensrisiko abstellt, nicht abgelehnt werden. ${ }^{4}$ Testet man - alternativ zum Jahr 2000/01 - das Jahr 2005/06 auf einen Strukturbruch in den Einkommensschwankungen, so führt dies zu qualitativ gleichwertigen Ergebnissen.

\section{Fazit und Ausblick}

Politikfolgenabschätzungen sind insbesondere auf dem Feld der Agrarpolitik hoch relevant und im Regelwerk der EU vorgegeben. Hierbei werden die Auswirkungen politischer Reformen der vergangenen Jahre auf die Einkommenshöhe, Überwälzungseffekte und das Einkommensrisiko in der Landwirtschaft seit längerer Zeit intensiv diskutiert. Umfangreichere empirische Untersuchungen auf Basis einzelbetrieblicher Daten lagen hierzu jedoch bislang nicht vor. Ziel dieser Untersuchung war es deshalb, mit Hilfe einer Paneldatenanalyse den Einfluss der wichtigsten EU-Agrarreformen sowie der Einführung des EEG auf die betriebliche Einkommenshöhe, die gezahlten Pachtpreise und das Einkommensrisiko zu analysieren. Hierzu wurden Deckungsbeitrags- und Pachtpreiszeitreihen für 22 nordrhein-westfälische Marktfruchtbetriebe für den Zeitraum von 1984/85 bis 2010/11 herangezogen. Mit der

4 Folgende alternative Vorgehensweisen für die Messung des Einkommensrisikos liefern qualitativ übereinstimmende Ergebnisse: Erstens, die absoluten Abweichungen der Deckungsbeiträge werden für alle drei Zeitabschnitte berechnet. Im Anschluss werden die Residuen im Rahmen eines Panelmodells analysiert. Zweitens, es werden für alle Betriebe Standardabweichungen der Deckungsbeiträge für die dre Zeiträume berechnet (also pro Betrieb drei Standardabweichungen) und diese dann im Rahmen eines Panelmodells verglichen. 
Analyse wird ferner ein Modellrahmen vorgeschlagen, der bei Vorliegen einzelbetrieblicher Daten beliebig reproduzierbar und damit z.B. auch auf zukünftige Reformen im Agrarbereich anwendbar ist.

Die vorliegende Analyse ist insbesondere für Politikberater für die adäquate Unterstützung politischer Entscheidungsträger bei Politikfolgenabschätzungen von hoher praktischer Relevanz. So zeigen die Ergebnisse, dass die Einkommen der betrachteten landwirtschaftlichen Betriebe durch die Verringerung der Interventionspreise und die Einführung gekoppelter Direktzahlungen im Zuge der MacSharry-Reform signifikant gesunken sind. Durch die Agenda 2000, die Fischler Reform und die Einführung des EEG konnte hingegen ein wiederum signifikant steigendes Einkommensniveau verzeichnet werden. Dies könnte unter anderem in den preissteigernden Effekten für Agrarrohstoffe im Allgemeinen begründet sein, welche durch die sich ausbreitende Energiemaisproduktion im Zuge des EEG bedingt wurden. Des Weiteren wurden bestehende Überwälzungseffekte auf dem Pachtmarkt durch die politischen Reformen beeinflusst. So stiegen die Pachtpreise für die betrachteten Betriebe mit der Einführung von Direktzahlungen im Zuge der MacSharry Reform signifikant an. Im Zusammenhang mit den sinkenden Einkommen für die Landwirte deutet dies darauf hin, dass der Wechsel von Preisstützungssystemen hin zu flächenbezogenen Direktzahlungen ceteris paribus einen Einkommenstransfer von den Landwirten hin zu den Landeigentümern bewirkt haben könnte. Außerdem lassen die Ergebnisse eine Verringerung des betrieblichen Einkommensrisikos durch die MacSharryReform, also durch die Verringerung der Interventionspreise und die Einführung von Direktzahlungen, vermuten. Dies erscheint auf den ersten Blick kontraintuitiv, könnte aber durch einen natürlichen Hedge mit den ebenfalls schwankenden Inputpreisen begründet sein. Dies könnte umgekehrt bedeuten, dass die betrieblichen Einkommen durch die Mindesteinspeisevergütungen in der sich ausweitenden Biogasproduktion in Zukunft stärker schwanken könnten, sofern die betreffenden Betriebe gleichzeitig keine Maßnahmen zur langfristigen Absicherung ihrer Inputpreise ergreifen.

$\mathrm{Zu}$ den Ergebnissen der vorliegenden Untersuchung muss einschränkend hinzugefügt werden, dass es seit der Jahrtausendwende neben den EU-Agrarreformen und der Einführung des EEG weitere Veränderungen in den allgemeinen Marktbedingungen gab, die die Höhe der betrieblichen Einkommen, der Pachtpreise und das Einkommensrisiko ebenfalls beeinflusst haben könnten. Hierzu zählt unter anderem das zunehmende Engagement außerlandwirtschaftlicher Investoren im Agrarbereich. In Bezug hierauf könnten ökonomische Experimente die vorliegende Untersuchung ergänzen. Diese haben gegenüber empirischen Vorgehensweisen den Vorteil, Daten unter kontrollierten Bedingungen zu erheben und somit die Auswirkungen der Veränderungen bestimmter Variablen (z.B. einem Politikwechsel) auf die Zielgröße (z.B. der Deckungsbeitragshöhe) ceteris paribus zu quantifizieren. Politikfolgenabschätzungen für konkrete zukünftige Reformvorhaben könnten somit zusätzlich verbessert werden. Des Weiteren wäre eine vertiefende Analyse der Fragestellung mit diversifizierteren landwirtschaftlichen Unternehmen von Interesse. Auch könnte eine größere Anzahl von Betrieben aus geographisch unterschiedlichen Regionen die vorhandenen Ergebnisse ergänzen.

\section{Literaturverzeichnis}

Arellano, M. (1987): PRACTITIONERS'CORNER: Computing Robust Standard Errors for Within-groups Estimators. In: Oxford Bulletin of Economics and Statistics 49: 431-434.

Baltagi, B. (2009): Econometric Analysis of Panel Data. 4. Auflage. Wiley, Chichester.

Benz, M. (2006): Entrepreneurship as a Non-profit-seeking Activity. In: International Entrepreneurship and Management Journal 5: 23-44.

Breusch, T. und A. Pagan (1979): A Simple Test for Heteroscedasticity and Random Coefficient Variation. In: Journal of the Econometric Society 47: 1287-1294.

Britz, W., A. Gocht, I.P. Domínguez, T. Jansson, S. Grosche und N. Zhao (2012): EU-weite (Regional- und Betriebsgruppen-) Effekte durch Prämienentkopplung und Harmonisierung in Folge der Health-CheckReform. In: German Journal of Agricultural Economics 61: 44-56.

Bundesbank (2012): VBI - Verbraucherpreisindex. Internet: http:// www.bundesbank.de.

Chatzis, A. (1997): Flächenbezogene Ausgleichszahlungen der EU-Agrarreform: Pachtmarktwirkungen und Quantifizierung der Überwälzungseffekte. In: Agrarwirtschaft, Sonderheft.

Christensen, L. und W. Greene (1976): Economies of Scale in U.S. Electric Power Generation. In: Journal of Political Economy 84: 655-676.

Ciaian, P., D. Kancs und J.F.M. Swinnen (2008): Static and Dynamic Distributional Effects of Decoupled Payments: Single Farm Payments in the European Union. In: LICOS Discussion Papers 207/2008. LICOS Centre for Institutions and Economic Performance, K.U. Leuven, Leuven. Daugbjerg, C. und A. Swinbank (2007): The Politics of CAP Reform: Trade Negotiations, Institutional Settings and Blame Avoidance. In: Journal of Common Market Studies 45: 1-22.

Europäische Kommission (1991): The development and future of the CAP. COM(91)100. CEC, Brüssel.

Europäische Kommission (2005): Council Regulation (EC) No 1698/2005. Official Journal of the European Union L, 277.

Gömann, H., P. Kreins und T. Breuer (2007): Deutschland - EnergieCorn-Belt Europas? In: German Journal of Agricultural Economics 56: 263-271.

Happe, K. und A. Balmann (2002): Struktur-, Effizienz- und Einkommenswirkungen von Direktzahlungen. In: German Journal of Agricultural Economics 51: 366-388.

Hausmann, J. (1978): Specification Tests in Econometrics. In: Econometrica 46: 1251-1271.

Hazell, P.B.R. (1971): A Linear Alternative to Quadratic and Semivariance Programming for Farm Planning under Uncertainty. In: American Journal of Agricultural Economics 53: 53-62.

Hsiao, C. (2003): Analysis of Panel Data. Cambridge University Press, New York.

Isermeyer, F. (2003): Umsetzung des Luxemburger Beschlusses zur EUAgrarreform in Deutschland: eine erste Einschätzung. Arbeitsbericht 3/2003. Johann-Heinrich von Thünen-Institut, Braunschweig.

Kilian, S. und K. Salhofer (2009): Entkoppelte Prämien, Bodenpreise und Wettbewerbsfähigkeit. In: German Journal of Agricultural Economics 58: 141-143.

Kreins, P. und H. Gömann (2008): Modellgestütze Abschätzung der regionalen landwirtschaftlichen Landnutzung und Produktion in Deutschland vor dem Hintergrund der "Gesundheitsüberprüfung “ der GAP. In: German Journal of Agricultural Economics 57: 195-206.

Morck, R. (2000): Concentrated Corporate Ownership. University of Chicago Press, Chicago. 
OECD (2010): The OECD-FAO Agricultural Outlook: 2010-2019. Organization for Economic Co-operation and Development, Paris.

Schmidt, E. (2005): Der Kommissionsvorschlag zur Reform der EUZuckermarktordnung: „Schnellschuss“ und „überzogene Reform“ oder sorgfältig erarbeitetes Konzept für den Einstieg in ein schrittweises „Ende des Sozialismus“ auch im Zuckersektor? In: German Journal of Agricultural Economics 54: 145-147.

Sckokai, P. und D. Moro (2006): Modeling the Reforms of the Common Agricultural Policy for Arable Crops under Uncertainty. In: American Journal of Agricultural Economics 88: 43-56.

Sckokai, P. und D. Moro (2009): Modelling the impact of the CAP Single Farm Payment on farm investment and output. In: European Review of Agricultural Economics 36: 395-423.

Simon, H.A. (1956): Rational Choice and the Structure of Environments. In: Psychological Review 63: 129-138.

Tangermann, S. (2011): Policy Solutions to Agricultural Market Volatility: A Synthesis. ICTSD 65, Genf.

Thompson, S.R., R. Hermann und W. Gohout (2000): Agricultural Market Liberalization and Instability of Domestic Agricultural Markets: The Case of the CAP. In: American Journal of Agricultural Economics 82: 718-726.

von Ledebur, E.O. und J. Schmitz (2011): Preisvolatilität auf landwirtschaftlichen Märkten. Arbeitsberichte aus der VTI-Agrarökonomie Nr. 05/2011, Internet: http://hdl.handle.net/10419/45374.

Wooldridge, J.M. (2010): Econometric analysis of cross section and panel data. 2. Auflage. MIT Press, Cambridge.

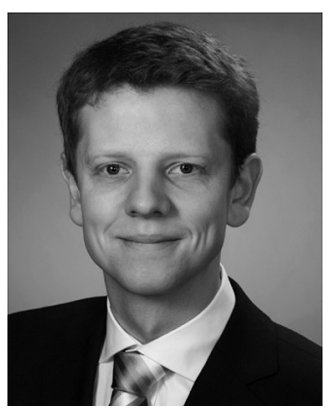

Dr. Jan-Henning Feil ist wissenschaftlicher Mitarbeiter am Arbeitsbereich Landwirtschaftliche Betriebslehre des Departments für Agrarökonomie und Rurale Entwicklung an der Georg-August-Universität Göttingen. Er studierte Betriebswirtschaftslehre und promovierte im Bereich Agrarökonomie ebenfalls an der Georg-August-Universität Göttingen. Seine Forschungsinteressen umfassen den Bereich der Investition und Finanzierung sowie die einzelbetriebliche Politikfolgenabschätzung. Email: jan-henning.feil@agr.uni-goettingen.de

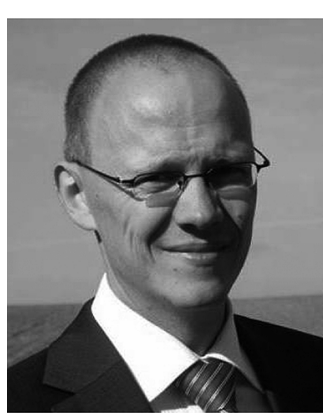

Prof. Dr. Oliver Mußhoff leitet den Arbeitsbereich Landwirtschaftliche Betriebslehre des Departments für Agrarökonomie und Rurale Entwicklung an der Georg-August-Universität Göttingen. Er studierte Agrarwissenschaften und promovierte im Bereich Agrarökonomie an der Humboldt-Universität zu Berlin. Seine Forschungsinteressen umfassen quantitative Planungsmethoden und die Analyse des unternehmerischen Entscheidungsverhaltens. Email: oliver.musshoff@agr.uni-goettingen.de

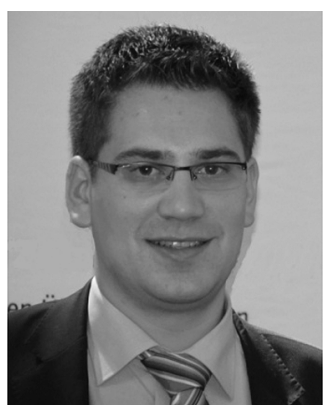

Tobias Roeren-Wiemers ist selbstständiger Landwirt im Kreis Paderborn. Er studierte Agrarwissenschaften an der Georg-August Universität Göttingen mit dem Schwerpunkt Wirtschafts- und Sozialwissenschaften des Landbaus. Email: Tobias@Roeren-Wiemers.de 